\title{
Effects of acupoint-stimulation for the treatment of primary dysmenorrhoea compared with NSAIDs: a systematic review and meta-analysis of 19 RCTs
}

Yang $\mathrm{Xu}^{1,2+}$, Wenli Zhao ${ }^{1,3+}, \mathrm{Te} \mathrm{Li}^{4}$, Huaien Bu${ }^{5}$, Zhimei Zhao ${ }^{6}$, Ye Zhao ${ }^{7,8^{*}}$ and Shilin Song ${ }^{9^{*}}$

\begin{abstract}
Background: Primary dysmenorrhoea (PD), defined as painful menses in women with normal pelvic anatomy, is one of the most common gynaecological syndromes. Acupoint-stimulation could potentially be an effective intervention for PD. Our aim was to determine the effectiveness of acupoint-stimulation compared with Non-Steroidal Anti-Inflammatory Drugs (NASIDs) in the treatment of PD.

Methods: Six databases were searched to December 2014. Sixteen studies involving 1679 PD patients were included. We included randomized controlled trials that compared acupoint-stimulation with NASIDs for the treatment of PD. The main outcomes assessed were clinical effectiveness rate, symptom score, visual analogue score, variation in peripheral blood prostaglandin F2a (PGF2a) and side effects. All analyses were performed using Comprehensive Meta-Analysis statistical software.
\end{abstract}

Results: (1) The total efficacy was better than control group: odds ratio $=5.57 ; 95 \%$ confidence interval $(95 \% \mathrm{Cl})=3.96,7$. 83; $P<0.00001$; (2) The effect of intervention was positive in relieving the severity of PD symptoms: mean difference $(M D)=2.99 ; 95 \% C l=2.49,3.49 ; P<0.00001 ;$ (3) No statistical difference existed between two groups in terms of a reduction in the VAS: $\mathrm{MD}=1.24 ; 95 \% \mathrm{Cl}=-3.37,5.85 ; P=0.60$; (4) The effect of intervention on the variation in peripheral blood PGF2a between two groups was positive: $\mathrm{MD}=7.55 ; 95 \% \mathrm{Cl}=4.29,10.82 ; P<0.00001$; (5) The side effects of control groups was more than the acupoint-stimulation group: $\mathrm{OR}=0.03 ; 95 \% \mathrm{Cl}=0.00,0.22 ; P=0.0005$.

Conclusions: According to this article, acupoint-stimulation can relieve pain effectively in the treatment of PD and offers advantages in increasing the overall effectiveness.

Keywords: Acupoint-stimulation, Primary dysmenorrhoea, Meta-analysis, Systematic review, Non-steroidal antiinflammatory drugs

\section{Background}

Dysmenorrhea is the most common gynecologic complaint among adolescent and young adult females. The prevalence of dysmenorrhoea appears to differ across the world, ranging from $80 \%$ in Western Australia [1], to 60\%

\footnotetext{
*Correspondence: zaky@ufl.edu; haiguagua@163.com

${ }^{\dagger}$ Equal contributors

7Department of Chemical Engineering, University of Florida, 1006 Center Drive, Gainesville, FL 32611, USA

'Laboratory of Anatomy, School of Integrative Medicine, Tianjin University of Traditional Chinese Medicine, No. 88 Yu Quan Road, Nankai District, Tianjin 300193, China

Full list of author information is available at the end of the article
}

in Canada [2], 48.4\% in Mexico [3], and 79.9\% in Iran [4]. Over $50 \%$ of females of reproductive age have painful menstruation; among them, $10 \%$ have severe dysmenorrhoea, whereby their monthly lives' quality is impaired from 1 to 3 days differently [5]. It starts some hours before menstruation and continues for up to 48-72 h, and takes the form of pains and cramps in the lower abdomen radiating towards the inner side of the thighs [6]. Half of such cases experience systemic symptoms, such as nausea, vomiting, diarrhoea, fatigue, irritability and dizziness $[7,8]$, which reduce the quality of life. The patients with mild-to-moderate pain can manage their pain without 
drugs or with a small amount of non-prescription drugs. However, approximately $15 \%$ of all women experience severe dysmenorrhoea to a level that affects work or study; such women need drugs to relieve their pain [9]. Dysmenorrhea in adolescents and young adults is usually primary, and is defined as painful menses in women with normal pelvic anatomy [10]. In 10\% of females with severe dysmenorrhea symptoms, pelvic abnormalities such as endometriosis or uterine anomalies may be found (secondary dysmenorrhea) [11]. This article mainly discusses primary dysmenorrhoea (PD).

In recent years, there are more and more researches about the pathogenesis of PD. In addition to factors relating to the body's nerve, genetic and immune systems, and psychological/social factors, the pathogenesis is generally considered to be mainly related to two factors: (1) abnormal uterine contraction, and (2) endocrine and metabolic factors. The state of uterine ischemia and hypoxia causes the uterine muscle to contract, increasing intrauterine tension, and so leading to abdominal pain. Patients with abnormal uterine contractions and the subjective feeling of abdominal colic have been consistently reported over time. Many types of molecular endocrine factors play an important role in the pathogenesis of PD, such as prostaglandins (PGs), oxytocin (OT) and vasopressin (VP), $\beta$-EPs, nitric oxide (NO), noradrenaline (NE), endothelins, and magnesium and calcium ions. In particular, prostaglandin F2 $\alpha$ (PGF2 $\alpha$ ), cyclooxygenase (COX) metabolite of arachidonic acid, causes potent vasoconstriction and myometrial contractions, leading to uterine ischemia and pain [12].

Treatment for PD includes a variety of pharmacological and non-pharmacological methods. Common pharmacological interventions include Non-Steroidal Anti-Inflammatory Drugs (NASIDs) and oral contraceptives. NSAIDs are widely used as the first-line therapy for females with dysmenorrhoea $[13,14]$. However, there are often adverse events associated with the use of NSAIDs, including stomach ache, diarrhoea, nausea, and liver or kidney damage after discontinuing medication [13]. Therefore, many patients with PD are seeking complementary and alternative techniques such as acupointstimulation to treat the symptoms of PD [15], which emphasizes stimulating the acupoint(s) to strengthen the body's endogenetic regulated function, so as to preventing and treating diseases by regulating the meridian system.

Although previously publications have reported that acupuncture-related treatments are effective for primary dysmenorrhea, the evidence is low convincing due to insufficient methodological quality and small sample size. Given the safety of acupoint-stimulation [16], therefore, the purpose of this systematic review and meta-analysis study is to determine the effectiveness of acupointstimulation in treating PD.

\section{Methods}

\section{Search strategy}

We searched six electronic databases that included PubMed, the Cochrane Library, Embase, the Chinese Academic Journals Full-text Database, the Chinese Science and Technology Journal Full-text Database (CNKI), Wanfang Data, and the Chinese Biomedical Literature Database (VIP). The index terms were the following: dysmenorrhoea, menorrhagia, painful menstruation, menstrual, pain, painful menstruation, menstrual pain, menstrual pains, acupuncture, moxibustion, auricular point, ear acupoint (administering persistent/ temporary pressure with Cowherb seed/finger force to stimulate pressure points), electroacupuncture, acusector, acupoint application, randomized controlled trials, controlled clinical trials, and random. The above terms in Chinese were adapted and searched in Chinese databases. The studies were published between the first year they were available and December 2014, which of the language is Chinese and English.

\section{Selection criteria and exclusion criteria Selection criteria}

- Research Type

- Research Subjects

- Interventions

- Outcomes (Clinical effectiveness rate, Symptom score, Visual analogue score, Peripheral blood PGF2 $\alpha$, Side effects)

\section{Research type}

Randomized controlled trials (RCTs).

\section{Research subjects}

Patients with a definite PD diagnosis: PD is defined as painful menses in women with normal pelvic anatomy. An eligible patient is diagnosed based on the PD Clinical Guideline of the Society of Obstetricians and Gynaecologists of Canada.

\section{Interventions}

Intervention groups - acupoint-stimulation, including acupuncture, moxibustion, ear acupressure, electroacupuncture, acupoint application; Control groups - NSAIDs.

\section{Outcomes}

(1) Clinical effectiveness rate

It was a dichotomous outcome and the overall effectiveness of acupoint-stimulation therapy as a subjective assessment, which was defined as the proportion of participants who got relieved pain and was based on response evaluation criteria used in the treatment of insomnia with acupoint-stimulation. What's more, it was 
PRISMA 2009 Flow Diagram

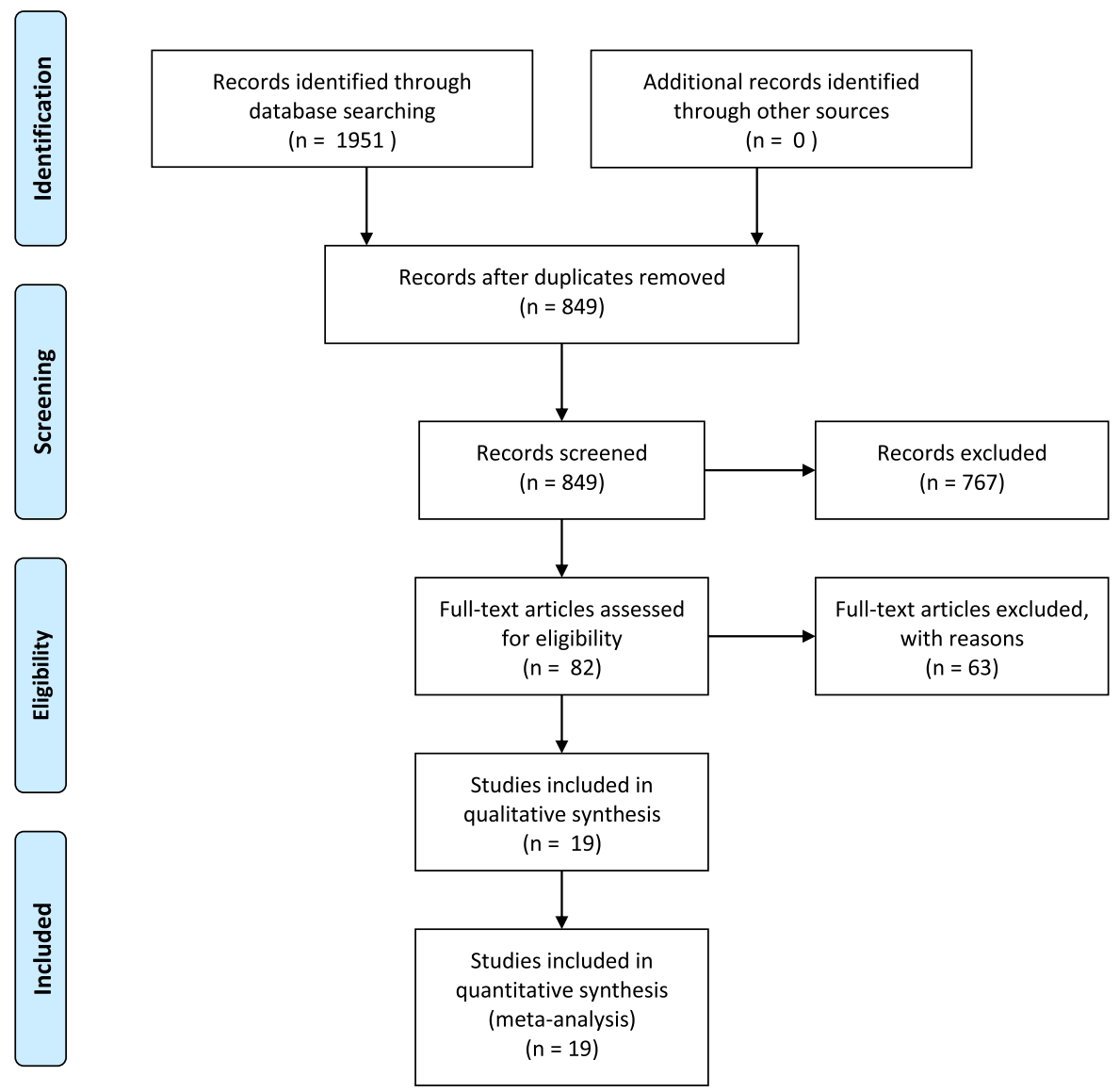

Fig. 1 PRISMA 2009 Flow Diagram

reported by trial participants themselves. For example, clinical therapeutic effect criteria were categorized as cure, markedly effective, effective, or ineffective. According to the Guideline for Clinical Trials of New Patent Chinese medicines (GCTNPCM) [17] evaluation standards, which define: Cured: after treatment, the score of symptoms was;restored to 0 , abdominal pain and other symptoms disappeared and the dysmenorrhea did not recurred 3 menstrual cycles after treatment; Markedly effective: after treatment, the score of symptoms was decreased to less than $1 / 2$ of the score before treatment, abdominal pain obviously relieved and other symptoms improved and the patient without taking analgesics could insist in work; Effective: after treatment,the score of symptoms decreased to $1 / 2-3 / 4$ of the score before treatment, abdominal pain relieved and other symptoms improved, and the patient could work after taking analgesics; Ineffective: abdominal pain and other symptoms did not change. The total number of "cure, markedly effective, effective" were used to calculate effective rate.

(2) Symptom score

In accordance with the GCTNPCM, the patients' symptom scores were recorded before and after treatment [18].

(3) Visual analogue score (VAS)

In the paper, we draw a $10 \mathrm{~cm}$ above the horizontal line and horizontal line of the end of 0 , indicating no pain; on the other side of 10 , said the pain; middle part of said varying degrees of pain. Feel the patient according to uniform mark on the horizontal line, indicating the degree of pain [19].

(4) Peripheral blood PGF2 $\alpha$.

The blood was taken from cubital vein within $24 \mathrm{~h}$ in the last menstrual period before treatment and within $24 \mathrm{~h}$ in the next menstrual period after treatment for one course, and the plasma PGF2 $\alpha$ levels in the two groups were determined with radioimmunoassay.

(5) Side effects 
Xu et al. BMC Complementary and Alternative Medicine (2017) 17:436

Page 4 of 12

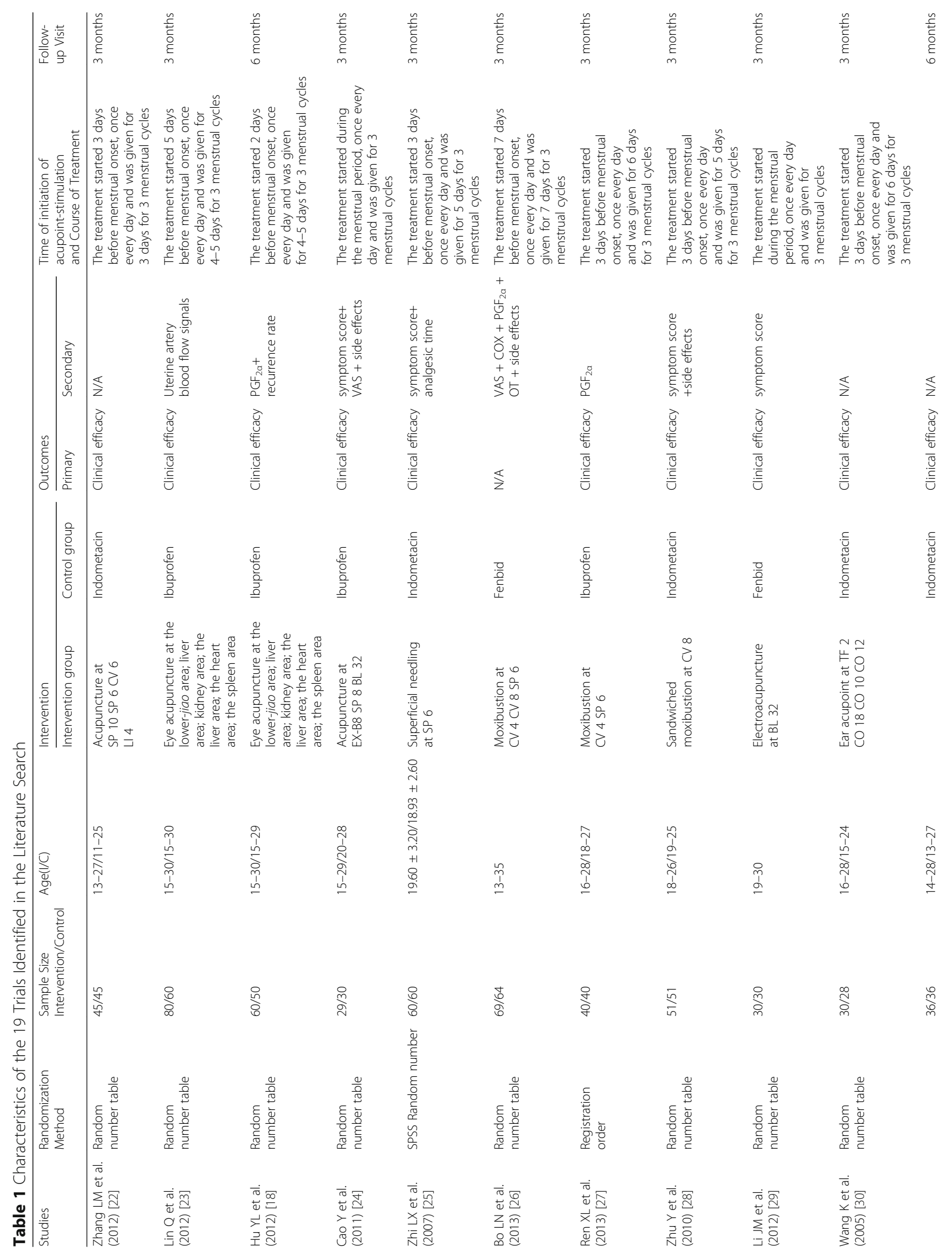


Xu et al. BMC Complementary and Alternative Medicine (2017) 17:436

Page 5 of 12

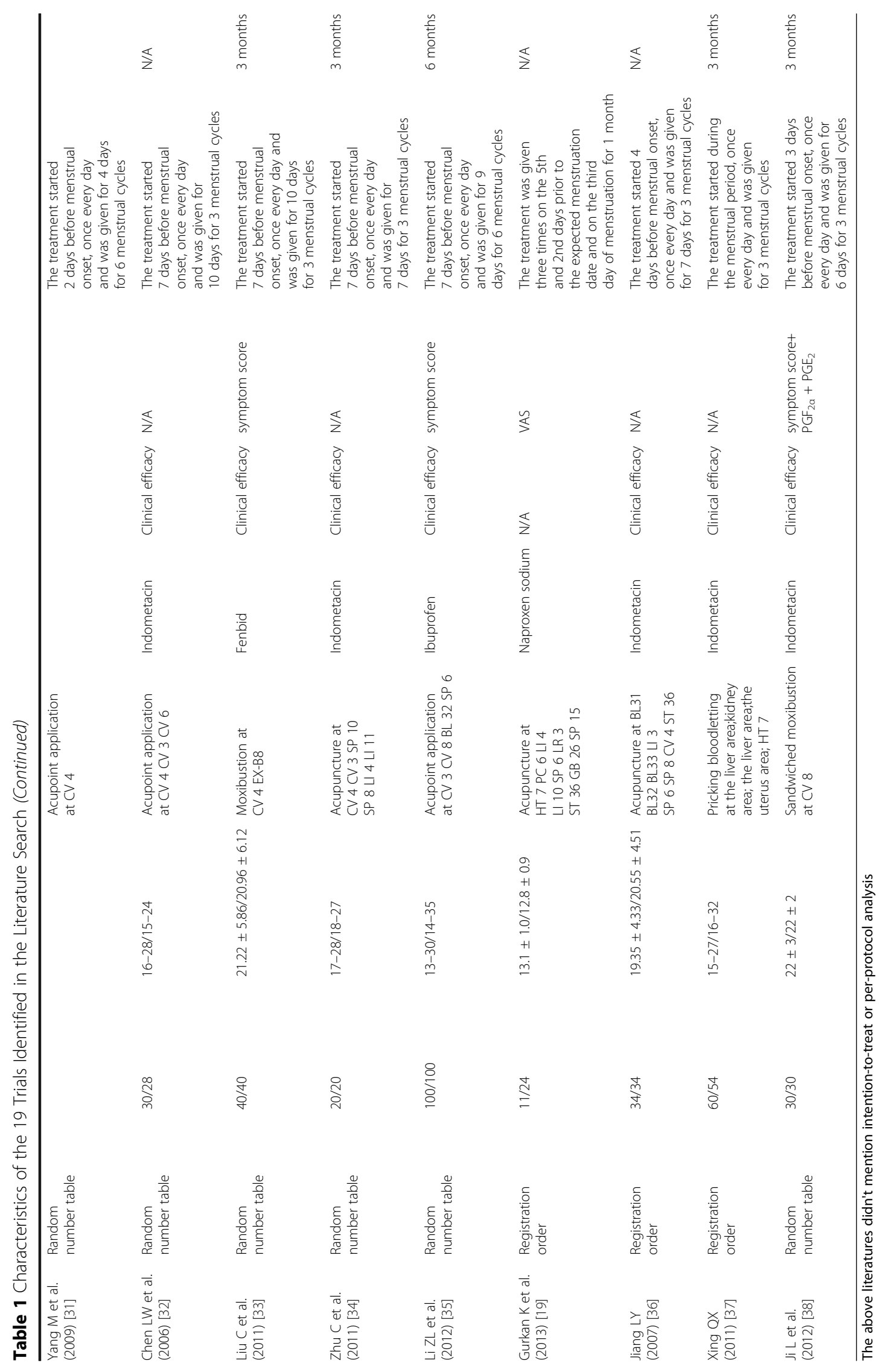




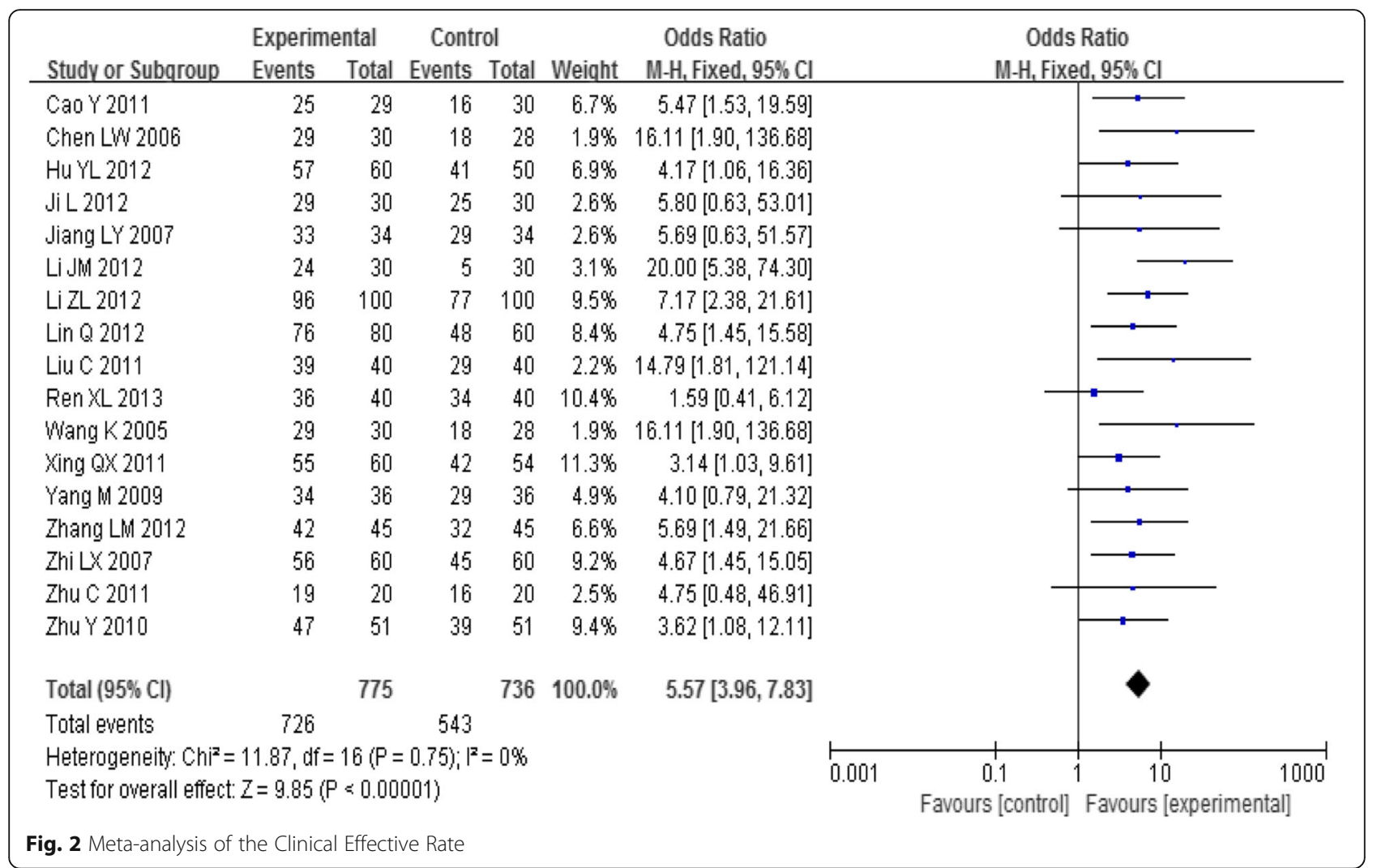

To observe the vital signs before and after treatment and whether there were fainting, stomach ache, diarrhoea, nausea, and liver or kidney damage during the treatment and other adverse events occurred, and recorded.

\section{Exclusion criteria}

(1)Trials where it was unclear whether a randomized trial was being conducted;
(2) Trials conducted using combinations of treatments and many medical interventions;

(3) Trials in which the data were inadequate and difficult to extract.

Data extraction and quality assessment

Searches were conducted and the data extracted by two independent researchers. Each trial identified in the search was evaluated for design, eligibility criteria for participants, and outcome measures. Any disagreement

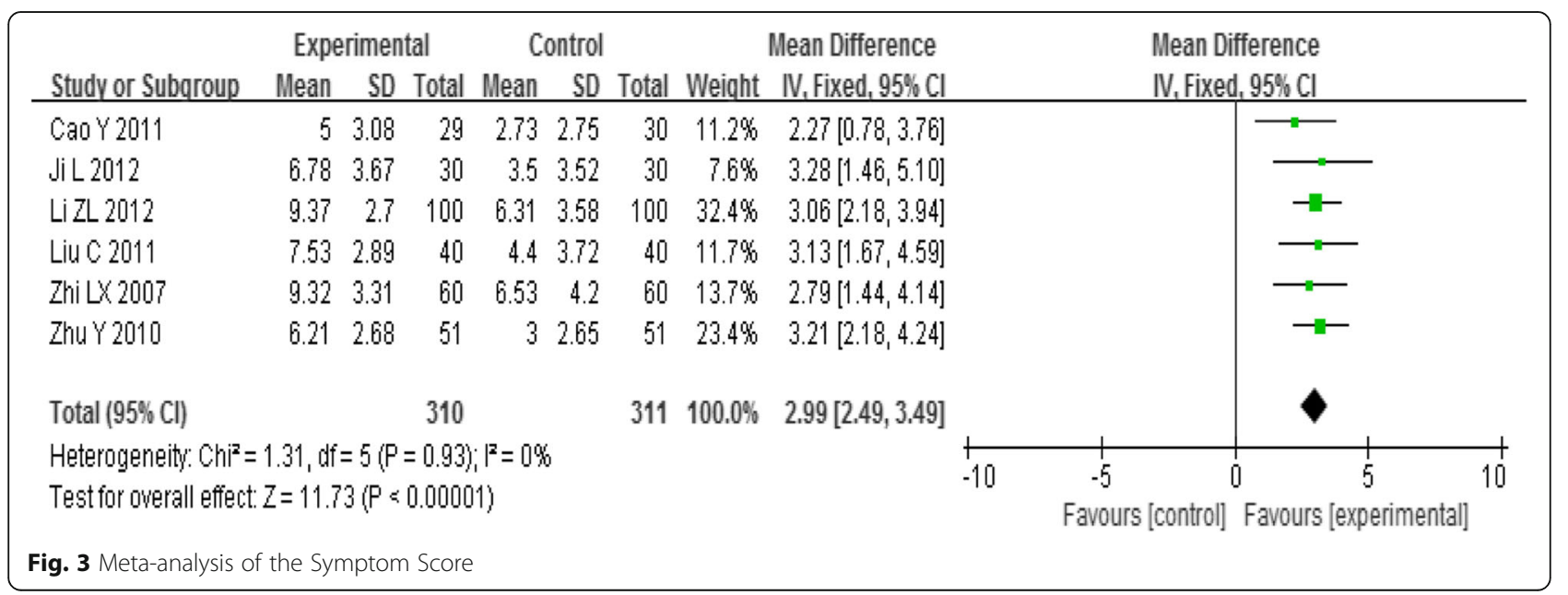




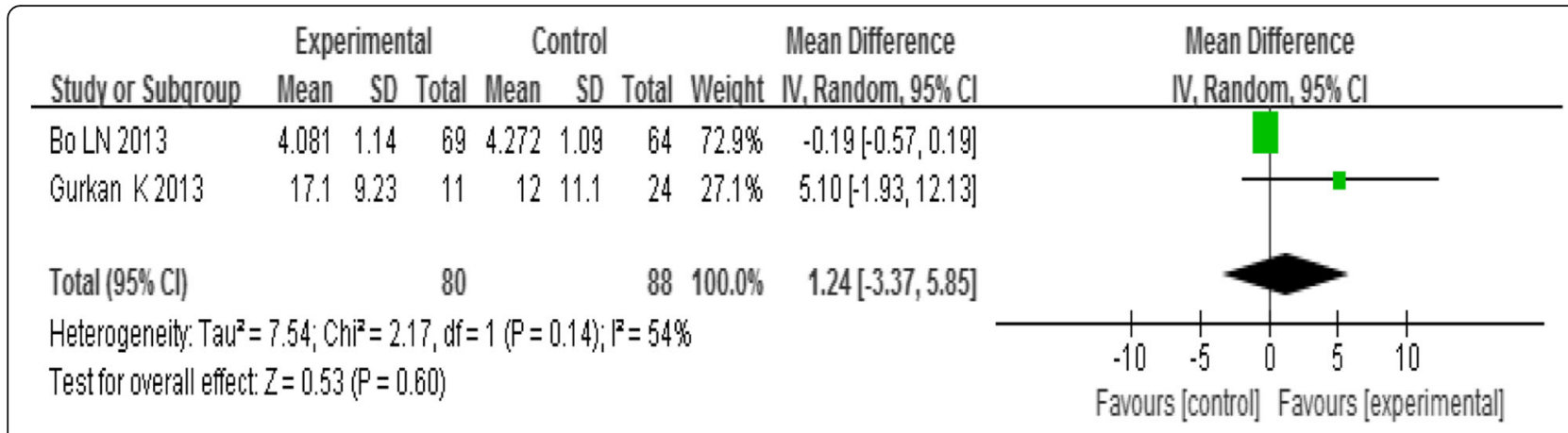

Fig. 4 Meta-analysis of the VAS

between researchers with regard to the eligibility of a trial was resolved by consulting a third researcher. We created a form for data extraction which included: (1) basic information about each trial, including the topic, first author, dateline and journal; (2) basic information about the patients, including the number of cases in each group and the mean age; (3) the study design and intervention; and (4) the outcomes.

The quality of the trials included in this study was assessed by other two researchers according to the Cochrane Handbook for Systematic Reviews of Interventions, Version 5.1.0.

\section{Statistical analyses}

All analyses were performed using Comprehensive MetaAnalysis statistical software, RevMan 5.1.0 (Cochrane Collaboration, Copenhagen, Denmark). Continuous outcome variables were analyzed using a standardized measure; dichotomous variables were compared and the results presented as odds ratios/risk ratios (OR/RR).

To obtain a standard deviation of the change from baseline for the experimental intervention, use $\left(R_{1}=0.5\right)$ [20]:

$$
S D(C)=\sqrt{S D(B)^{2}+S D(F)^{2}-\left(2 \times R_{1} \times S D(B) \times S D \times(F)\right)}
$$

$S D(B)$ represents the standard deviation before intervention; $S D(F)$ represents the standard deviation after intervention.
The research team evaluated homogeneity among the trials via $\mathrm{I}^{2}$. If $\mathrm{I}^{2}$ was $\geq 50 \%$, the trials were considered to be heterogeneous, and a random-effect model based on a Mantel-Haenszel (MH) or inverse variance (IV) statistical approach was selected. If $\mathrm{I}^{2}$ was $<50 \%$, the studies were considered to be homogeneous, and a fixed-effects model based on an MH or IV statistical approach was used. Pooled summary statistics of the differences in the ratio or mean of the individual studies were developed. Pooled differences in ratios or means, and two-sided $P$ values were calculated and used as criteria for determining the level of statistical significance. $P<0.05$ was considered to indicate statistical significance. Moreover, a sensitivity analysis was conducted based on the leaveone-out cross-validation procedure [21].

\section{Results}

Study selection

A flow chart of the included/excluded studies is shown in Fig. 1. Database searches yielded 70 studies from PubMed, 28 from the Cochrane Central Register of Clinical Trials, 215 from Embase, 552 from CNKI, 328 from Wanfang Data, 279 from VIP, and 479 from CBM. After removal of duplicate records, 849 records remained. Following the first review based on the title, 149 records were remained, and the abstracts were reviewed based on the pre-defined eligibility criteria. A total of 82 records were selected for full text review and data processing. During this

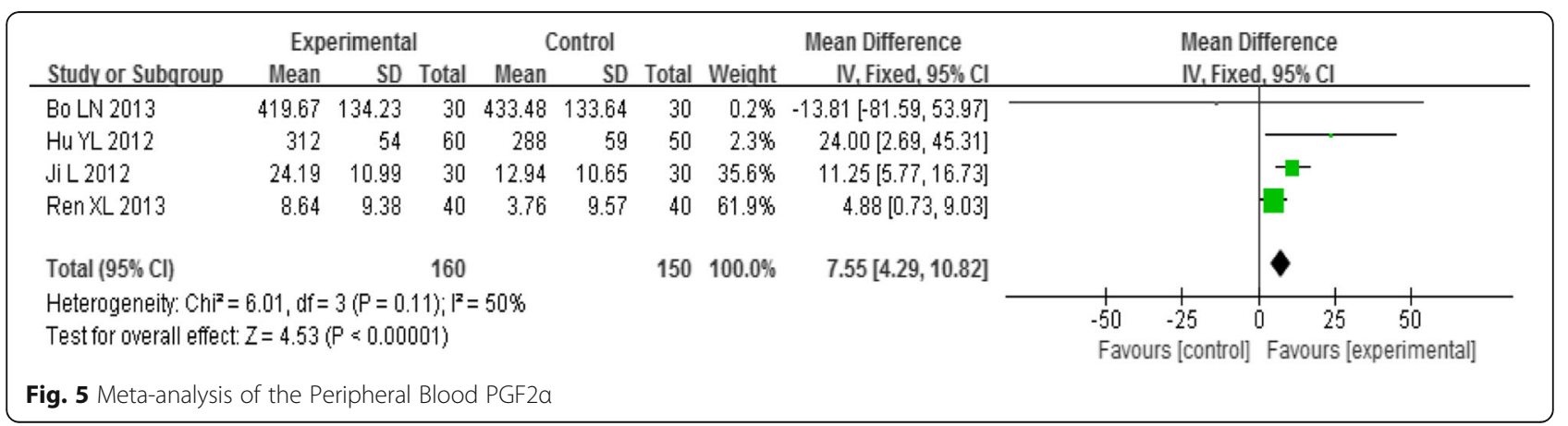




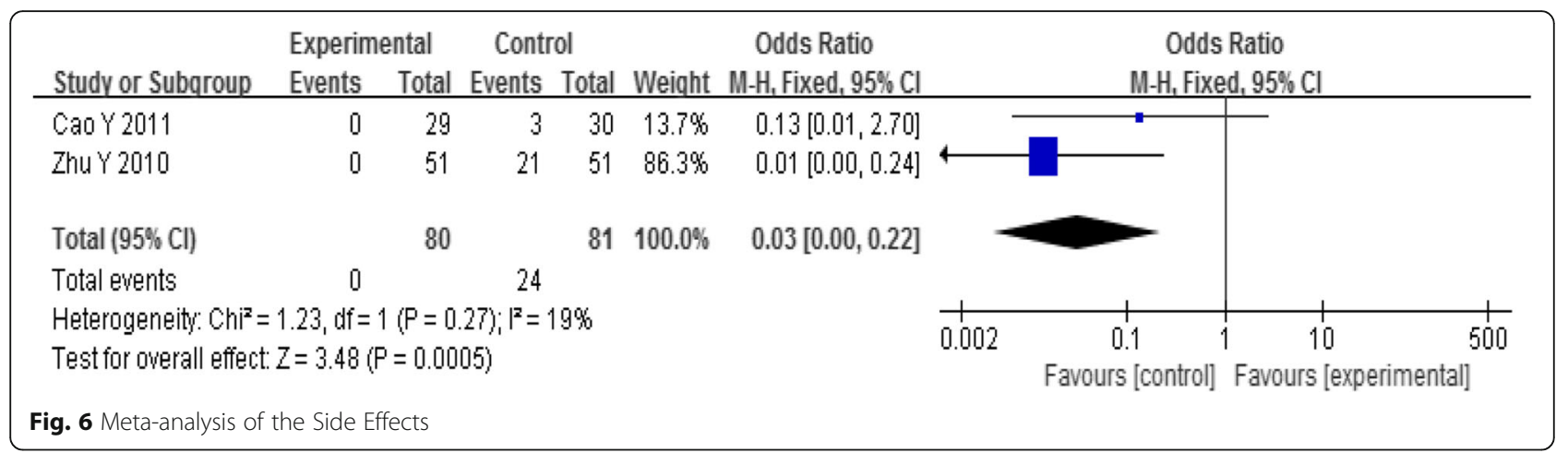

phase, 63 papers were excluded, so 19 studies were included in the final meta-analysis, comprising 1679 participants.

\section{Characteristics of the included studies}

Table 1 shows the main characteristics of the 19 RCTs [18, 19, 22-38].

\section{Clinical outcomes \\ Clinical effectiveness rate}

Seventeen trials examined the effects of acupointstimulation and reported the clinical effectiveness rate of treatment for participants with PD who used those therapies versus the rate for a control group. Analysis of pooled data using a fixed-effect model showed that the effect of intervention on the clinical effectiveness rate was positive $[\mathrm{OR}=5.57,95 \% \mathrm{CI}(3.96,7.83), P<0.00001]$ (Fig. 2). That is to say, the clinical effectiveness rate, the acupointstimulation group being superior to the NSAIDs.

\section{Symptom score}

Six trials reported the symptom score. Analysis of pooled data using a fixed-effect model showed that the effect of intervention on the symptom score was positive $[\mathrm{MD}=2.99,95 \% \mathrm{CI}(2.49,3.49), P<0.00001]$ (Fig. 3). The curative effect of acupoint-stimulation on PD is significant.

\section{visual analogue score}

Three trials reported the VAS; analysis of pooled data using a random-effect model showed that $\mathrm{I}^{2}=98 \%$, indicating heterogeneity. So, the trial by Cao (2011) was excluded from analysis, then analysis of the pooled data using a random-effect model showed that there was no statistical difference in variation of VAS between the groups receiving acupoint-stimulation and the control groups $[\mathrm{MD}=1.24,95 \% \mathrm{CI}(-3.37,5.85), P=0.60]$ (Fig. 4).

\section{Peripheral blood PGF2a}

Four trials examined the effects of acupoint-stimulation and reported peripheral blood PGF2 $\alpha$ of participants with PD who used those therapies versus the rate for a control group. Analysis of the pooled data using a fixedeffect model showed that the effect of intervention on the variation in peripheral blood PGF2 $\alpha$ between the groups receiving acupoint-stimulation and the control groups was positive $[\mathrm{MD}=7.55,95 \% \mathrm{CI}(4.29,10.82)$, $P<0.00001$ ] (Fig. 5). In the study, it is indicated that acupoint-stimulation can effectively decrease peripheral blood PGF2 $\alpha$ level in the patient of PD, so as to inhibit PGF2 $\alpha$-induced spastic contraction of uterine muscle, improve the decrease of blood flow, and relieve the symptoms of the patient of dysmenorrhea.

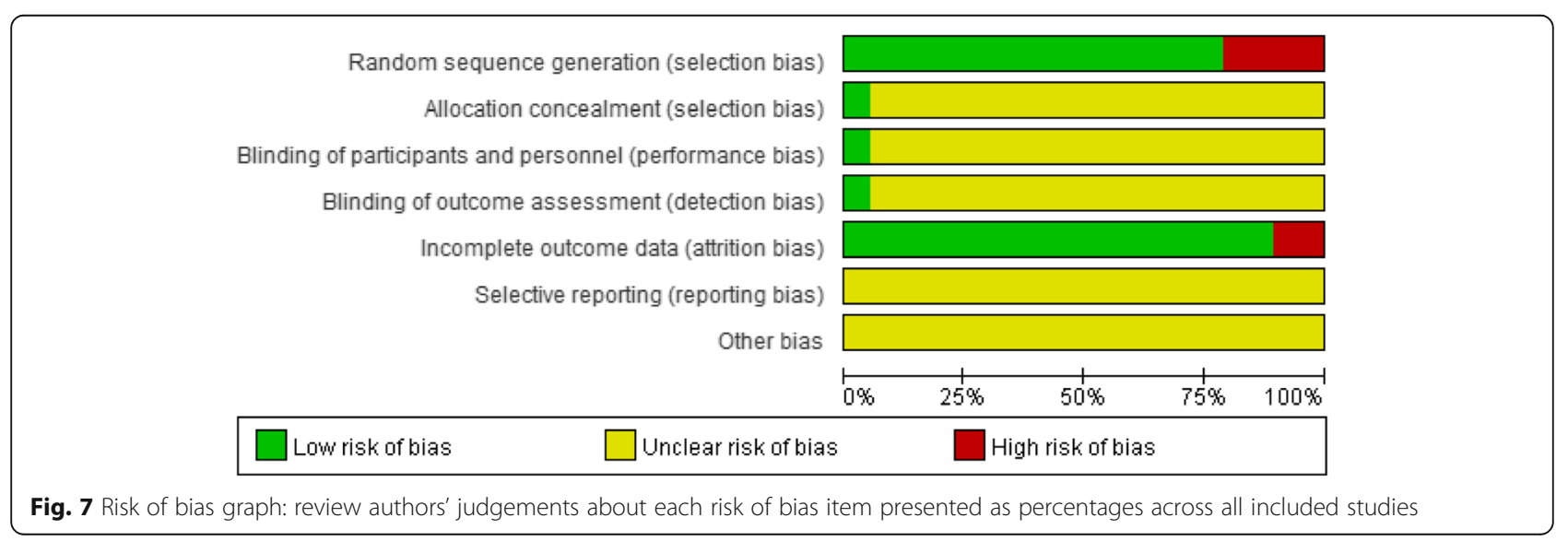




\section{Side effects}

Three trials reported the side effects between acupointstimulation and control group. Analysis of pooled data using a random-effect model showed that $\mathrm{I}^{2}=83 \%$, indicating heterogeneity. So the trial by Bo (2013) was excluded from analysis, then analysis of the pooled data using a fixed-effect model showed that the side effects of control groups were more than the acupoint-stimulation group. [OR $=0.03$, 95\%CI $(0.00,0.22), P=0.0005]$ (Fig. 6).

\section{Quality assessment}

The risks of seven biases among the 19 trials were evaluated, including random sequence generation, allocation concealment, blinding of participants and personnel, blinding of outcome assessment, incomplete outcome data, selective reporting, and other biases according to the criteria in the Cochrane Handbook for Systematic Reviews. Fifteen of the studies described correct randomization methods. There was only one trial with allocation concealment and blinding of participants and personnel and blinding of outcome assessment, and nearly all of the trials failed to mention allocation concealment, the blinding of the participants and personnel, and the blinding of outcome assessments. The methodological qualities of the included trials are summarized in Figs. 7 and 8.

\section{Funnel plot of publication bias}

The research team performed an analysis of all the included studies, using a funnel plot to determine publication bias in all of the literature. The outcome from the funnel plot analysis is summarized in Fig. 9. The outcome suggests that there was little publication bias.

\section{Discussion}

\section{Meta-analysis of clinical effect}

In the 19 RCTs included, 17 reported a clinical effectiveness rate and 6 reported symptom scores and 4 reported variation in the level of PGF $2 \alpha$ in the peripheral blood of women with PD. The meta-analysis revealed that acupoint-stimulation is superior to NSAIDs in the treatment of PD in terms of clinical effectiveness rate and symptom improvement and reducing the concentration of PGF $2 \alpha$ in peripheral blood.

Only one RCT reported uterine artery blood flow signals. The results showed that the uterine arterial pulsation index (PI) and uterine arterial resistance index (RI) of the dysmenorrheal patients were significantly increased in the eye acupuncture group before treatment. Moreover, most studies used a subjective, self-reported index of treatment effects as the outcome measure. Because participants self-reported without additional objective outcomes, their pain status could not be assessed accurately [39]. Furthermore, the included studies used

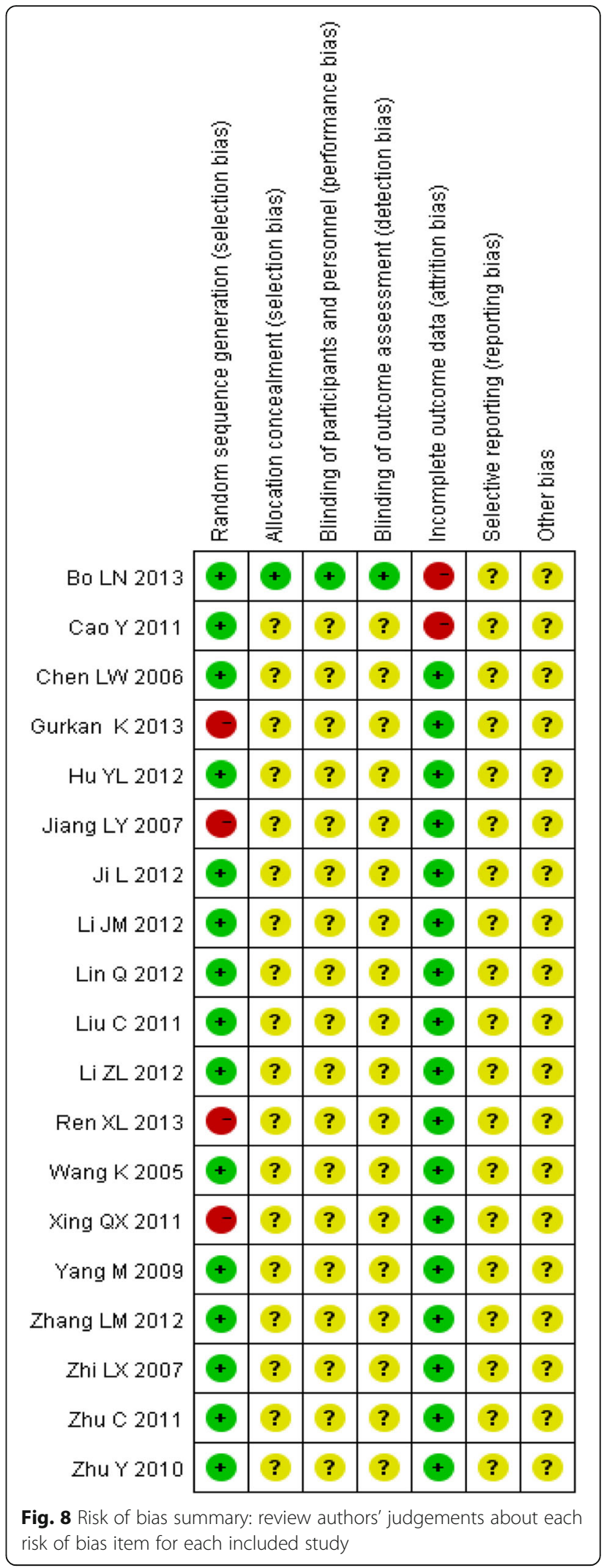




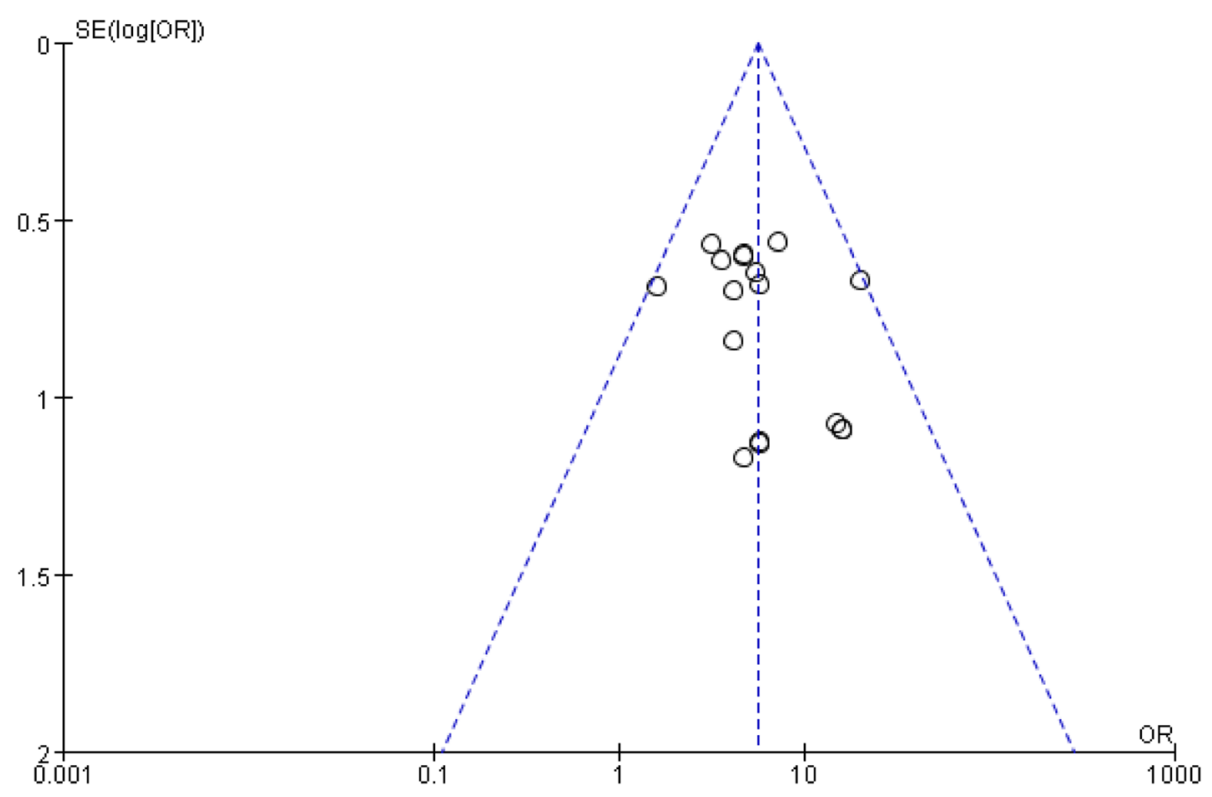

Fig. 9 Total Effects of Intervention Groups

different treatments for the intervention group, such as acupuncture, moxibustion, ear acupressure, electroacupuncture and acupoint application, which of the purpose is to highlight the specificity of acupoints.

\section{The reason for NSAIDs being a drug of positive control}

NSAIDs act by inhibiting the enzyme that catalyzes the conversion of arachidonic acid to cyclic endoperoxides, namely COX, which in turn inhibits the production of PGs [40, 41]. The resulting lower levels of PGs lead to less vigorous contractions of the uterus, and therefore to less discomfort. Thus, NSAIDs alleviate primary dysmenorrheic pain predominantly through the suppression of endometrial PGs synthesis [42]. Although NSAIDs is the first-line treatment for PD, it also has shortcomings, which can inhibit the synthesis of $\mathrm{COX}-1$, as well as COX-2, finally it is easy to cause adverse reactions of gastrointestinal and central nervous system. Vane [43] indicated in 1994 that the effective treatment effect of NSAIDs was due to inhibition of COX-2, however, the adverse reactions imputed the suppression of COX-1. Therefore, we consider that NSAIDs may be used as a drug of positive control.

Although the results are encouraging, the conclusions from the current study should be carefully considered before being applied to clinical practice specific patients especially individuals with NSADIs contraindication. This study aims to collect all RCTs relating to acupointstimulation treatment of PD and use systematic review to gauge the effectiveness of acupoint-stimulation in the treatment of PD in order to use this treatment more widely in clinical practice.

\section{Different conclusions of the published literature}

Some evidence indicates that acupoint-stimulation is effective in treating primary dysmenorrhea [44-49], but that evidence was largely based on one small, randomized, controlled trial. However, two more recent sham acupuncture randomized controlled trials failed to show evidence of pain reduction $[50,51]$. One of the major challenges may be the subjective nature of the symptoms' presentations and acupoints utilized. Although a few reviews $[15,21,52,53]$ of acupuncture for the treatment of PD are currently available, none of those reviews analyze the potential mechanism of acupuncture for the treatment of PD, which is the key research content in future. Therefore, a systematic review with a meta-analysis is necessary so that quality evidence can be put forward for the use (or not) of acupointstimulation for the treatment in individuals with PD.

\section{Limitations and strengths}

The limitations of this evaluation system are as follows: (1) most of the researches did not mention how the sample size was estimated, and most sample sizes were small, leading to a low inspection efficiency; (2) in some of the studies there was inadequate reporting of allocation concealment; implementing or not fully implementing allocation concealment will lead to an exaggerated curative effect; (3) the results were heterogeneous on account of their use of subjective indicators to evaluate the curative effect (symptom scores, VAS), so that implementation of the blinding method is important, but the included studies did not describe the implementation of the blinding method; (4) the study was limited to Chinese and 
English research, leading to the possibility of selection bias, and the terminology or the guidelines used in clinical managements might not be in the same language.

The strengths of this evaluation system are as follows: this is the first report that comparing the effect of acupoint-stimulation and NSADIs in the treatment of $\mathrm{PD}$, and it provides new evidence and open new horizons that acupoint-stimulation can relieve pain effectively in the treatment of PD and offers advantages in increasing the overall effectiveness.

\section{Perspectives}

In our future research, we will conduct some trials relating to acupoint-stimulation for the treatment of PD, which will focus on the following aspects to prevent bias: (1) an estimation of sample size, (2) a fully random design incorporating allocation concealment, and (3) a blind design for the proposer, performer and measurer.

\section{Conclusion}

The current evidence reveals that acupoint-stimulation in the treatment of PD has some obvious advantages compared with treatment by NSADIs. The advantages are that acupoint-stimulation can alleviate the symptoms of dysmenorrhoea, reduce the level of peripheral blood PGF2 $\alpha$ and has fewer side effect, so it can be used to treat PD patients, especially individuals with NSADIs contraindication.

\begin{abstract}
Abbreviations
Cl: Confidence interval; CNKI: Chinese Science and Technology Journal Fulltext Database; COX: Cyclooxygenase; COX-2: Cyclooxygenase 2;

GCTNPCM: The Guideline for Clinical Trials of New Patent Chinese Medicines; IV: Inverse variance; MD: Mean difference; MH: Mantel-Haenszel;

NE: Noradrenaline; NSAIDs: Non-Steroidal Anti-Inflammatory Drugs; OR: Odds ratios; OT: Oxytocin; PD: Primary dysmenorrhoea; PGF2a: Prostaglandin F2a; PGs: Prostaglandins; PI: Pulsation index; PRC: People's Republic of China; RCTs: Randomized controlled trials; RI: Resistance index; RR: Risk ratios; TCM: Traditional Chinese Medicine; VAS: Visual analogue score; VIP: Chinese Biomedical Literature Database; VP: Vasopressin
\end{abstract}

\section{Acknowledgments}

Medical writing services were provided by YiHeAn Biotechnology Co. Ltd. Authors retained full control of manuscript content.

\section{Funding}

This project was supported by the Science Foundation on Traditional Chinese Medicine/Integrative Medicine of the Tianjin Health and Family Planning Commission (2015036).

\section{Availability of data and materials}

Data are all contained within the paper.

\section{Authors' contributions}

W.L.Z., Y.Z. And S.L.S conceived and designed the review; Y.X. And W.L.Z analyzed the data; H.E.B. Contributed reagents/materials/analysis tools; Z.M.Z., W.L.Z. And T.L. wrote the paper. All authors read and approved the final manuscript.

\section{Authors' information}

All authors contributed to the design and concept, performed the searches required for their assigned sections, wrote a section, read, revised and critiqued the successive versions, and approved the final manuscript. YZ coordinated the effort and integrated the sections and comments.
Ethics approval and consent to participate

N/A.

Consent for publication

Not applicable.

Competing interests

The authors declare that they have no competing interests.

\section{Publisher's Note}

Springer Nature remains neutral with regard to jurisdictional claims in published maps and institutional affiliations.

\section{Author details}

${ }^{1}$ Graduate School, Tianjin University of Traditional Chinese Medicine, Tianjin 300193, China. ${ }^{2}$ Department of Gynecology and Obstetrics, Nankai Hospital, Tianjin Academy of Integrative Medicine, Tianjin 300100, China. ${ }^{3}$ Department of Neurology, Nankai Hospital, Tianjin Academy of Integrative Medicine, Tianjin 300100, China. ${ }^{4}$ Department of Chinese Medicine, Tianjin Hearing Impairment Specialist Hospital, Tianjin 300150, China. ${ }^{5}$ Department of Public Health, School of Chinese Medicine, Tianjin University of Traditional Chinese Medicine, Tianjin 300193, China. ${ }^{6}$ Department of Gynecology and Obstetrics of Chinese Medicine, First Teaching Hospital of Tianjin University of Traditional Chinese Medicine, Tianjin 300193, China. ${ }^{7}$ Department of Chemical Engineering, University of Florida, 1006 Center Drive, Gainesville, FL 32611, USA. ${ }^{8}$ Institute for Cell \& Tissue Science and Engineering, University of Florida, Gainesville, FL 32611, USA. 'Laboratory of Anatomy, School of Integrative Medicine, Tianjin University of Traditional Chinese Medicine, No. 88 Yu Quan Road, Nankai District, Tianjin 300193, China.

Received: 7 November 2016 Accepted: 11 August 2017

Published online: 31 August 2017

\section{References}

1. Hillen TIJ, Grbavac SL, Johnston PJ, Straton JAY, Keogh JMF. Primary dysmenorrhea in young western Australian women: prevalence, impact, and knowledge of treatment. J Adolesc Health. 1999;25(1):40-5.

2. Burnett MA, Antao V, Black A, Feldman K, Grenville A, Lea R, Lefebvre G, Pinsonneault O, Robert M. Prevalence of primary dysmenorrhea in Canada. J Obstet Gynaecol Can. 2005;27(8):765-70.

3. Ortiz Ml, Rangel-Flores E, Carrillo-Alarcón LC, Veras-Godoy HA. Prevalence and impact of primary dysmenorrhea among Mexican high school students. Int J Gynecol Obstet. 2009;107(3):240-3.

4. Jalili Z, Safizadeh H, Shamsipoor N. Prevalence of primary dysmenorrhea in college students in Sirjan. Kerman Payesh. 2005;4(1):61-7.

5. Dawood MY. Dysmenorrhea. Clin Obstet Gynecol. 1983;26(3):719-27.

6. Jun EM, Chang S, Kang DH, Kim S. Effects of acupressure on dysmenorrhea and skin temperature changes in college students: a non-randomized controlled trial. Int J Nurs Stud. 2007:44(6):973-81.

7. Speroff L, Fritz MA. Clinical Gynecologic Endocrinology and Infertility (7th edition). Philadelphia: Lippincott Williams and Wilkins, 2005, Menstrual disorders; pp. 401-464

8. Balbi C, Musone R, Menditto A, Prisco LD, Cassese E, D'Ajello M, et al. Influence of menstrual factors and dietary habits on menstrual pain in adolescence age. Eur J Obstet Gynecol Reprod Biol. 2000;91(2):143-8.

9. Ylikorkala O, Dawood MY. New concepts in dysmenorrhea. Am J Obstet Gynecol. 1978;130(7):833-47.

10. Pu BC, Fang L, Gao LN, Liu R, Li AZ. Animal study on primary dysmenorrhoea treatment at different administration times. Evid Based Complement Alternat Med. 2015;2015:367379.

11. Harel Z. Dysmenorrhea in adolescents and young adults: from pathophysiology to pharmacological treatments and management strategies. Expert Opin Pharmacother. 2008;9(15):2661-72.

12. Alvin PE, Litt IF. Current status of etiology and management of dysmenorrhea in adolescents. Pediatrics. 1982;70(4):516-25.

13. Dawood MY. Nonsteroidal anti-inflammatory drugs and changing attitudes toward dysmenorrhea. Am J Med. 1988;84(5):23-9.

14. Kaplan Ö, Nazıroğlu M, Güney M, Aykur M. Non-steroidal anti-inflammatory drug modulates oxidative stress and calcium ion levels in the neutrophils of patients with primary dysmenorrhea. J Reprod Immunol. 2013;100(2):87-92. 
15. Yang H, Liu CZ, Chen X, Ma LX, Xie JP, Guo NN, Ma ZB, Zheng YY, Zhu J, Liu JP. Systematic review of clinical trials of acupuncture-related therapies for primary dysmenorrhea. Acta Obstet Gynecol Scand. 2008;87(11):1114-22.

16. Lin JG, Chen YH, Gao XY, Lao L, Lee H, Litscher G. Clinical efficacy, mechanisms, and safety of acupuncture and moxibustion. Evid Based Complement Alternat Med. 2014;2014:356258.

17. Zheng X-Y. Guideline for clinical trials of new patent Chinese medicines. 1st ed. Beijing: Ministry of Health of the People's Republic of China; 1993. p. 263-5.

18. $\mathrm{Hu} Y \mathrm{Y}$, Lin $\mathrm{Q}, \mathrm{Li}$ Y, Zheng XM. Effect of eye acupuncture on plasma PGF 2a in patients of primary dysmenorrhea. World J Acupuncture-Moxibustion. 2012; 22(1):17-22.

19. Kiran G, Gumusalan Y, Ekerbicer HC, Kiran H, Coskun A, Arikan DC. A randomized pilot study of acupuncture treatment for primary dysmenorrhea. Eur J Obstet Gynecol Reprod Biol. 2013;169(2):292-5.

20. Higgins JP, Green S. Cochrane Handbook for Systematic Reviews of Interventions (Version 5.1.0). The Cochrane Collaboration, 2011, Available from: http://www.cochrane.org/handbook. Accessed 17 July 2015.

21. Xu T, Hui L, Juan YL, Min SG, Hua WT. Effects of moxibustion or acupoint therapy for the treatment of primary dysmenorrhea: a meta-analysis. Altern Ther Health Med. 2014;20(4):33-42.

22. Zhang LM, Yang HY. The 45 cases on single acupuncture treatment for primary dysmenorrhea. Fujian J TCM. 2012;43(2):25-6.

23. Lin $\mathrm{Q}$, Chen WZ, Li Y, Hu YL. Effect of eye acupuncture on uterine artery blood flow in patients with primary dysmenorrhea. Shanghai J Acupunct Moxibustion. 2012;31(12):885-7.

24. Cao Y. Acupuncture clinical analysis of primary dysmenorrhea. Master Thesis Heilongjiang Chin Med College 2011: 26-29.

25. Zhi LX. Randomized controlled study on superficial needling for treatment of primary dysmenorrhea. Zhongguo Zhen Jiu. 2007;27(1):18-21.

26. Bo LN. A literature mining and randomized controlled trial of moxibustion in treating primary dysmenorrhea. Doctor Thesis Chengdu Chin Med College 2013: 43-60, 67-70.

27. Ren XL. Clinical study on the treatment of primary Dysmenorrhea with moxibustion. Global Traditional Chin Med. 2013;6(6):431-2.

28. Zhu Y, Chen RL, Le Jl, Miao FR. Efficacy observation of primary dysmenorrhea treated with isolated-herbal moxibustion on Shenque (CV8). Zhongguo Zhen Jiu. 2010;30(6):453-5

29. Li JM. Clinical study of electro-acupuncture treatment of primary dysmenorrhea Ci Liao acupoints. Master Thesis. Guangzhou Chin Med College. 2012: 15-17.

30. Wang K, Pan WY, Duan YH. Clinical study on the treatment of primary dysmenorrhea with auricu1ar acupuncture. Guangdong Med J. 2005;26(12):1728-30.

31. Yang $M$. The clinical observation of 36 cases on dysmenorrhea moxibustion treatment of primary dysmenorrhea. Hainan Med J. 2009;20(7):226-7.

32. Chen LW. The clinical observation of primary dysmenorrhea treated with the Chinese native medicine sticks on the acupuncture point. Master Thesis Guangzhou Chin Med College 2006: 20-22.

33. Liu C, Zhang HY. Therapeutic effect of moxibustion on primary dysmenorrhea due to damp-cold retention. World J Acupuncture-Moxibustion. 2011;21(3):1-4.

34. Zhu C. Clinical study on the treatment of haemorrheological nature blood stasis type of primary dysmenorrhea with moxibustion. Hubei J TCM. 2011;33(1):65-6.

35. Li ZL, Li YQ, Pan FQ, Bian WH, Chu JZ, Zhu PQ. Clinical observation on Chinese herbs acupoint of Yugui wenjing decoction stick to treat haemorrheological nature blood stasis type of primary dysmenorrhea. Modern J Integrated Traditional Chinese Western Med. 2012;21(5):483-4

36. Jiang LY. Clinical experience of acupuncture for treatment of 34 cases in primary dysmenorrhea. J Emerg Tradit Chin Med. 2007;16:620-1.

37. Xing QX. Observation on the therapeutic effect of pricking bloodletting at ear points on primary dysmenorrhea. Shanghai Zhenjiu Zazhi. 2011;30:235-6.

38. Ji L, Chen RL, Deng PY, Zhou LJ, Do SC, Zhu Y. Treatment effect of herbpartitioned moxibustion for dysmenorrhea of cold stagnation type and its effect on PGF2a and PGE2. Shanghai J Acupunct Moxibustion. 2012;31:882-4.

39. Sale H, Hedman L, Isberg A. Accuracy of patients' recall of temporomandibular joint pain and dysfunction after experiencing whiplash: a prospective study. J Am Dent Assoc. 2010;141(7):879-86.

40. Warner TD, Giuliano F, Vojnovic I, Bukasa A, Mitchell JA, Vane JR. Nonsteroid drug selectivities for cyclo-oxygenase-1 rather than cyclo-oxygenase-2 are associated with human gastrointestinal toxicity: a full in vitro analysis. Proc Natl Acad Sci U S A. 1999:96(13):7563-8.
41. Ruoff G, Lema M. Strategies in pain management: new and potential indications for COX-2 specific inhibitors. J Pain Symptom Manag. 2003:25(2):S21-31.

42. Dawood MY. Dysmenorrhea. J Reprod Med. 1990;33(1):168-78.

43. Vane JR. Towards a better aspirin. Nature. 1994;36(2):215-6.

44. Dawood MY. Primary dysmenorrhea: advances in pathogenesis and management. Obstet Gynecol. 2006;108(2):428-41.

45. Doty E, Attaran M. Managing primary dysmenorrhea. J Pediatr Adolesc Gynecol. 2006;19(5):341-4.

46. Proctor M, Farquhar C. Diagnosis and management of dysmenorrhea. BMJ. 2006;332(7550):1134-8.

47. Sanfilippo J, Erb T. Evaluation and management of dysmenorrhea in adolescents. Clin Obstet Gynecol. 2008;51(2):257-67.

48. French L. Dysmenorrhea. Am Fam Physician. 2005:71(2):285-91.

49. Durain D. Primary dysmenorrhea: assessment and management update. Jidwifery Women's Health. 2004:49(6):520-8.

50. Smith CA, Crowther CA, Petrucco O, Beilby J, Dent H. Acupuncture to treat primary dysmenorrhea in women: a randomized controlled trial. Evid Based Complement Alternat Med. 2011;2011:612464.

51. Kempf D, Berger D, Ausfeld-Hafter B. Laser needle acupuncture in women with dysmenorrhoea: a randomised controlled double blind pilot trial. Forsch Komplementmed. 2009;16(1):6-12.

52. Chung YC, Chen $\mathrm{HH}$, Yeh ML. Acupoint stimulation intervention for people with primary dysmenorrhea: systematic review and meta-analysis of randomized trials. Complement Ther Med. 2012;20(5):353-63.

53. Abaraogu UO, Tabansi-Ochuogu CS. As acupressure decreases pain, acupuncture may improve some aspects of quality of life for women with primary Dysmenorrhea: a systematic review with meta-analysis. J Acupunct Meridian Stud. 2015:8(5):220-8.

\section{Submit your next manuscript to BioMed Central and we will help you at every step:}

- We accept pre-submission inquiries

- Our selector tool helps you to find the most relevant journal

- We provide round the clock customer support

- Convenient online submission

- Thorough peer review

- Inclusion in PubMed and all major indexing services

- Maximum visibility for your research

Submit your manuscript at www.biomedcentral.com/submit
) Biomed Central 